\begin{tabular}{ccc}
\hline & International Journal of Engineering \& Technology, $7(2.12)(2018) 145-146$ \\
SPC & International Journal of Engineering \& Technology \\
Website: $w w w . s c i e n c e p u b c o . c o m / i n d e x . p h p / I J E T$ & Research Paper \\
\hline
\end{tabular}

\title{
Methods of ship trajectory data processing for applying artificial neural network in port area
}

\author{
Kwang Il Kim ${ }^{1}$, Keon Myung Lee ${ }^{1 *}$, Jang Young Ahn ${ }^{2}$ \\ ${ }^{1}$ Dept. of Computer Science, Chungbuk National University, Cheongju, 28644, Republic of Korea \\ ${ }^{2}$ College of Ocean Science, JejuNational University, Jeju, 63243, Republic of Korea \\ *Corresponding author E-mail: kmlee@cbnu.ac.kr
}

\begin{abstract}
Background/Objectives: In Vessel Traffic Service (VTS), prediction of the flow of vessel traffic is essential to serve safety information and control ship traffic. However, it is difficult to predict a ship's speed due to many external forces and environmental conditions. This study proposes a data processing method to convert ship speed data to categorical data by dividing ship navigating routes into several gate lines.

Methods/Statistical analysis: A ship's trajectory is converted to each route's gate line speed. To determine the gate line speed, we converted the previous and subsequent gate line speeds into category data. The input and output category data were applied to a multilayer perceptron network using as input variables the previous speed variance category, ship type, and ship length, and as output variable the subsequent speed variance.

Findings: These results are useful because categorical data can be applied to various neural network models. As a result of the conducted experiments, the accuracy of the model improved when many gate lines are included.

Improvements/Applications: The study results can be applied to predict ship traffic flow for VTS operators.
\end{abstract}

Keywords: Vessel Traffic Service; Categorical data; Route Gate Line; Neural network; Automatic Identification System

\section{Introduction}

The Vessel Traffic Service (VTS), run by the International Maritime Organization, has been used in major port areas to serve safety information and control ship traffic. Under the VTS system, it is essential to accurately predict the flow of vessel traffic and transmit this information properly. Because a ship navigating in harbor water areas is affected by tidal currents, wind, waves, and other regulations related to harbor speed, it is difficult to accurately predict ship speeds.

Ship traffic data, such as radar and Automatic Identification System (AIS) data, have been collected by the relevant authorities. Using these AIS data, it is possible to perform assessments and predictions that will contribute to ship safety. Many researchers studied on forecasting maritime traffic flows using time series analysis. Mohamed $^{1}$ forecast the Suez Canal traffic converted into tonnage using ARIMA(Autoregressive Integrated Moving Average) and neural network models. It used continuous variable as output with multilayer perceptron. The ARIMA model has a slightly better performance than neural network model.

In recent years, neural network approach has been proposed for prediction of maritime traffic. This model is one of the non-linear model; input and output variables of neural network need to convert discrete or categorical type. Andrius ${ }^{2}$ preprocess ship's latitude and longitude data into turning regions using Density-based Spatial Clustering of Applications with Noise (DBSCAN) algorithm. They used ship's position cluster, ship ID, ship type and size as input data and turning region cluster as output data. Lokukalugeet $\mathrm{al}^{3}$ proposed the method to detect and track multiple-vessel movements using neural network.

The long-term accumulated AIS trajectory data are highly valued for use in machine learning areas, such as artificial neural networks including deep learning9. To apply an artificial neural network, a sufficient dataset and category response variables are required. In this study, we propose a method to convert ship speed data to categorical data by dividing ship navigating routes into several gate lines. Experiments were used to verify the model's accuracy and were conducted using multiple input and output variables.

The remainder of this paper is organized as follows. Section 2 describes the target data and explains its preprocessing. In Section 3 , the estimation route speed pattern using a neural network is presented. Section 4 describes experiments using an artificial neural network. Finally, conclusions are drawn in Section 5.

\section{Calculation of Route Gate Data}

Ships with more than 300 gross tonnage or those carrying passengers are equipped with an AIS device that broadcasts shipmovement information and receives other ships' traffic information ${ }^{4}$. The VTS center is a shore-based station that automatically collects information about ship movements via an AIS device. This AIS device transmits messages containing dynamic and static information in accordance with the update rate. Static data, including the ship's name, type, length, width, and draft information is transmitted every $6 \mathrm{~min}$ or upon request from a shore station. The dynamic data consist of the position, course, speed, 
heading direction, rate of turn, etc. of a ship, and these data are transmitted every $3-180 \mathrm{~s}$.

Because ship trajectory data from AIS are discrete sequence data, the ship trajectory points must be interpolated to calculate the gatepassing speed ${ }^{5}$. The interpolation method can be divided into two methods based on whether the difference of course $(\Delta d)$ is more than $5^{\circ}$.

In the case that $\Delta d$ is less than $5^{\circ}$, the ship's GPS positions (latitude, longitude) are defined as $x_{k}$ and $y_{k}$. Using two adjacent position sets [ $\left.\left(x_{k}, y_{k}\right),\left(x_{k+1}, y_{k+1}\right)\right]$ with subinterval $\left[x_{k} \leq x \leq x_{k+1}\right]$, a piecewise linear-interpolation position $\left(P_{i}(t)\right)$ can be determined with the formula ${ }^{6}$ :

$P_{i}(t)=y_{k}+\left(x_{k}-x_{i}\right) \frac{y_{k+t}-y_{k}}{x_{k+t}-x_{k}}$

In the case that $\Delta d$ is more than $5^{\circ}$, which is the curved-trajectory case, a cubic Hermite interpolation polynomial based on a cubic interpolation is applied to theship's course and speed sections ${ }^{5}$ as the formula:

$$
\begin{aligned}
S\left(x_{k}\right)= & \frac{-\left(-\Delta x \Delta m+2\left(\Delta y-\Delta x m_{k}\right)\right)}{\Delta x^{3}}\left(x-x_{k}\right)^{3}+ \\
& \frac{-\Delta x \Delta m+3\left(\Delta y-\Delta x m_{k}\right)}{\Delta x^{3}}\left(x-x_{k}\right)^{2}+m_{k}\left(x-x_{k}\right)+y_{k}
\end{aligned}
$$

Where $m_{k}$ slope of ship trajectory, $\Delta m=\left(m_{k+1}-m_{k}\right)$

A ship's speed data have the same time period to evenly separate the data. Therefore, we overlaid route gate lines in the water area. The route gates were created by dividing a ship's route into evenly spaced adjacent waypoints with gate length $d$ and width $w$. The gate-passing speed $\left(V_{i}\right)$ is calculated by dividing each gate length by the time difference between $G t_{i}$ and $G t_{i+1}$.

\section{Estimation Route Speed Pattern using Neural Network}

In this study, to determine the speed of a gate line in a ship's trajectory, we considered the previous speed change as the input variance $\left(d V I_{(i, j)}\right)$ and the subsequent speed change as the output variance $\left(d V O_{(i, j)}\right)$. These values were obtained by subtracting the previous and subsequent gate line speeds. Then, $d V I_{(i, j)}$ and $d V O_{(i, j)}$ were convertedto categorical valuesvia a five-step process. Figure 2 shows a schematic of how the speed variances at a gate line were determined $^{12}$

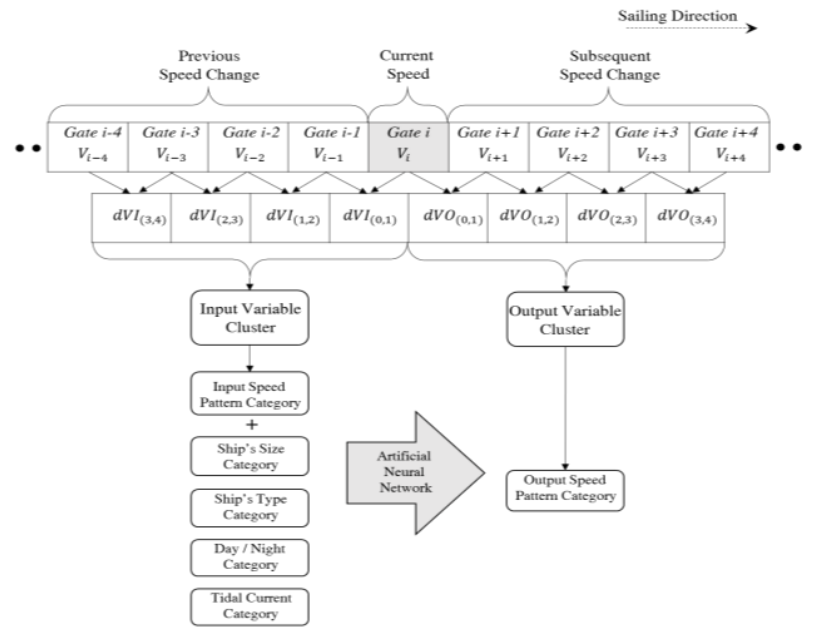

Fig. 2: Extraction of Model Variables from Route Gates.

To simplify the input and output data set, the input or output data were combined in a sequence dataset (input: $\left\{d V I_{1}, d V I_{2}, d V I_{3}, d V I_{4}\right\}$, output: $\left.\left\{d V I_{1}, d V I_{2}, d V I_{3}, d V I_{4}\right\}\right)$. After sorting each sequence dataset, a category number was granted if the frequency of the sequence dataset was greater than the threshold value. Table 1 represents the results of sequence dataset filtering with a threshold of 200 .
To train the artificial neural network, the input and output category data generated in Table 1 was applied to a multilayer perceptron neural network. Its input variables were the previous speed variance $\left(d V I_{1 \sim 4}\right)$ category, ship type, and ship length; the output variable was the subsequent speed variance $\left(d V O_{1 \sim 4}\right)$ with five hidden layers and a sigmoid activation function.

\section{Experiments}

The target water area is geographically surrounded by many islands. Many cargo and passenger ships enter or depart from port facilities through designated route passages 13 . For these experiments, we analyzed a ship's AIS data for one year and created 41,641 ship trajectory cases. Figure 3 shows the gate lines and distribution of ship trajectories in the Mokpo water area.

By applying the preprocessing methods to the ship trajectory data, input and output variables were calculated for the neural network. Experiments were performed by separating input variables from one to seven cases and output variable from one to five cases. The model accuracy of these experiments was evaluated using an additional one month's worth of actual data. Figure 4 shows the model accuracy for several cases.

\section{Conclusions}

The prediction of ship traffic flow is important to VTS operators. Currently operators predict ship traffic flow using a ship's present speed and distance remaining to be traveled. This method does not accurately take into account external forces, such as tidal currents and wind, when predicting the motion of multiple ships.

To apply an artificial neural network, sufficient data and category response variables are required. As previously mentioned, the neural network needs to convert a ship's movement variance to a categorical variable. In this paper, we proposed a method to convert a ship's speed data to categorical variables using a sequence dataset. Our method divided a ship's navigating routes into several gate lines. Then, experiments conducted using multiple input and output variables were used to verify the model's accuracy.

\section{Acknowledgment}

This research was supported by Next-Generation Information Computing Development Program through the National Research Foundation (NRF) of Korea (Grant no.: NRF-2017M3C4A7069432), by the MSIT(Ministry of Science and ICT), Korea, under the ITRC(Information Technology Research Center) support program(IITP2017-2013-0-00881) supervised by the IITP(Institute for Information \& communications Technology Promotion).

\section{References}

[1] Mohamed M., Forecasting the Suez Canal traffic: a neural network analysis, Maritime Policy \& Management, 2014, 31 (2), pp.139-156.

[2] AndriusDaranda. A Neural Network Approach to Predict Marine Traffic. Vilnius University Institute of Mathematics and Informatics, 2016.

[3] Lokukaluge P., Paulo Oliveira, and C. GuedesSoares, Maritime Traffic Monitoring Based on Vessel Detection, Tracking, State Estimation, and Trajectory Prediction.IEEE Transactions on Intelligent Transportation Systems, 2012 September, 13 (3), pp.1188-1200.

[4] Kim K. and Lee K., Ship Encounter Risk Evaluation for Coastal Areas with Holistic Maritime Traffic Data Analysis, Advanced Science Letters, 2017 October, 23 (10), pp.9565-9569.

[5] Kim K., Jeong J. and Park G., Analysis of Marine Accident Probability in Mokpo Waterways, Journal of Navigation and Port Research International Edition, 2011, 35 (9), pp.729-733.

[6] Lee K.,Cluster validity evaluation for small number of clusters, International Journal of Applied Engineering Research, 2014, 19 (21), pp.8933-8940. 